\title{
Reporting the small bowel examination
}

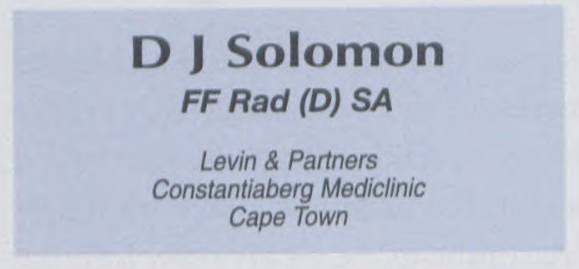

\section{Introduction}

Relative to the upper gastrointestinal tract and colon, diseases of the small intestine are relatively rare. Outside of a dedicated gastrointestinal unit requests for investigation of the small bowel are infrequent, limiting the interest and diligence of the radiologist performing the small bowel examination. Ultrasound, CT and more recently MR all contribute to assessment of small bowel pathology but generally the initial request is for a barium small bowel examination.

\section{Technique}

Many small bowel examinations are performed by conventional follow-through, with or without intermittent fluoroscopy during transit of barium through the small bowel. This procedure is limited by the narrowing of the lumen caused by drug-induced transit acceleration, causing crowding of the mucosal folds and a false appearance of nodularity. It cannot test distensibility of the lumen, and early mural infiltration or subtle stenoses may be missed. The small bowel enema (SBE) should be the investigation of choice.

Discomfort caused during intubation must be appreciated and the radiologist should be sensitive to the patient's need for reassurance, adequate topical anaesthesia and mild sedation if required.

The hypotonia induced by jejunal distention allows for display of all distended loops to the end of the examination and folds are straightened, making it possible to determine morphological normality. The examination is quicker and importantly more frequent fluoroscopy may show intermittent segmental dilatation, indicating subtle stenoses (Fig. 1).

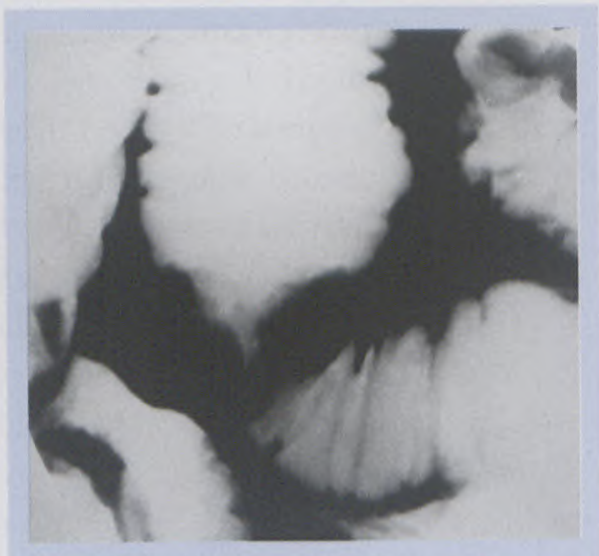

Fig.1. Intermittent segmental dilatation in a patient with multiple stenoses due to small bowel lymphoma.

\section{The report}

Interpreting and reporting the small bowel investigation depends on a knowledge of normal morphology and understanding the clinical history. Morphologic normality is based on observation of the fold pattern (valvulae conniventes), lumen diameter and wall thickness.

Fold density is higher in the jejunum (4 - 7 folds per inch) and gradually reduces to the ileum ( $2-4$ folds per inch).

Fold thickness is greater in the jejunum $(2 \mathrm{~mm})$, being thinner and shallower in the ileum (1 mm). It is important to measure fold thickness during adequate lumen distention. Lumen diameter is somewhat technique-dependent but generally considered to be up to $4.5 \mathrm{~cm}$ in the jejunum and $3.5 \mathrm{~cm}$ in the ileum. Wall thickness must be assessed when two adjacent loops are parallel to each other over a length of $5 \mathrm{~cm}$ and should be approximately $2 \mathrm{~mm}$ for a single wall thickness. Transit time through the small bowel is variable and may be influenced by factors such as infusion rate, presence of food content and a loaded caecum. Objective criteria have been defined but should only be applied when the infusion rate can be controlled by a mechanical pump. Assesment is made after $300 \mathrm{ml}$ have been infused at $75 \mathrm{ml} / \mathrm{min}$, with normal motility regarded as the jejunum having filled with approximately one-third of loops in contraction, hypermotility when greater than two-thirds of loops are in contraction, and hypomotility when the jejunum has not yet filled and few loops are in contraction. However, few radiologists have the use of a mechanical infusion pump and transit time is generally made subjectively.

As with all radiological investigations, reporting a study should depend on the clinical indication for the procedure and the questions asked by the referring doctor. The commonest indications for a small bowel examination are: $(i)$ assessment of Crohn's disease; (ii) diarrhoea or malabsorption states; (iii) partial mechanical small bowel obstruction; and (iv) obscure GIT bleeding. 


\section{Crohn's disease}

To confirm the presence or absence of Crohn's disease on the basis of history or prior studies requires a dedicated and detailed examination of the terminal ileum, assessing it in varying degrees of distension and compression. A normal report may state: 'Normal small bowel mucosal and fold patterns. The terminal ileum has a normal appearance and no features to suggest Crohn's disease have been demonstrated'. Having decided that the examination is abnormal the following features need to be mentioned in the report.

1. Are the abnormal features specific for Crohn's disease, and if not a differential diagnosis should be given. If the changes are non-specific it is important not to make a definitive radiological diagnosis of Crohn's as the label may remain with the patient for life. (Fig. 2).

2. Describe the nature and estimated length of involvement.

3. Identify and characterise proximal skip lesions.

4. Does the appearance of a stenosis suggest active inflammation which will respond to medical treatment or is it a smooth tapered fibrotic stricture with proximal dilatation that may require surgery.

5. Identify complications such as a fistula or features to suggest an adjacent abscess.

Each of these questions should be answered in the report as they have an important bearing on the clinician's choice of management.

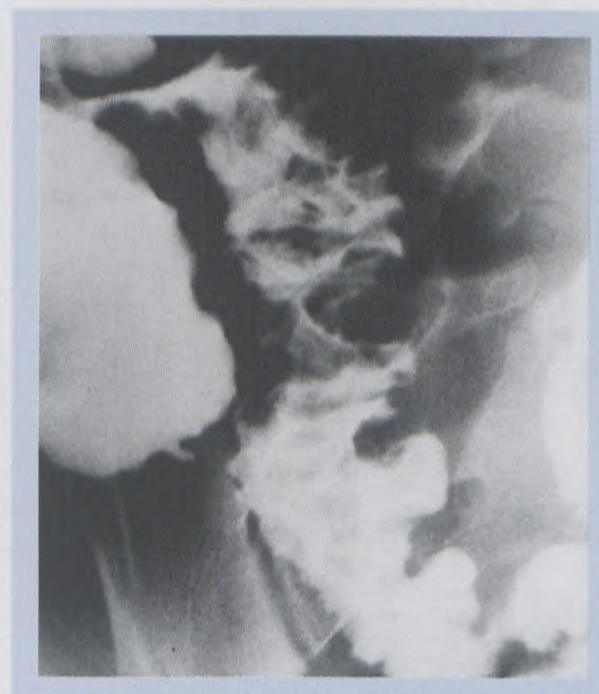

Fig. 2. Non-specific nodular mucosal swelling in the terminal ileum due to Yersinia enteritis.

\section{Diarrhoea/mal- absorption states}

Most pathological conditions of the small bowel that cause malabsorption are due to some form of mucosal infiltrate although other conditions such as lymphangectasia, jejunal diverticulosis, scleroderma and coeliac disease should be considered.

Therefore the following points should be reflected in the report of a small bowel examination in this clinical setting: (i) are there diverticulae? (ii) are the folds thickened, and if so is this regular or nodular, segmental or diffuse?; (iii) abnormality of fold density; and (iv) bowel shortening.

\section{Partial mechani- cal small bowel obstruction}

Here the aim is to identify stenotic lesions in the small bowel. Stenoses usually have a non-specific appearance but the likely cause may be inferred from the characteristics of the stenosis and the patient's history. The following features may help to narrow down the differential diagnosis: (i) the site of the stenosis; (ii) single or multiple stenoses; (iii) degree of proximal dilatation; and (iv) other small bowel abnormalities, in particular an abnormal terminal ileum which may indicate Crohn's disease.

\section{GIT bleeding}

The sensitivity of the small bowel examination in this clinical setting is low but a SBE is usually requested before proceeding to angiography. The report in this situation should reflect the following points: $(i)$ is there a Meckel's diverticulum? (ii) mucosal mass and its nature, e.g. a cavitating lesion may represent melanoma, lymphoma or leiomyosarcoma whereas a polypoid lesion may reflect a benign polyp or a melanoma metastasis; and (iii) focal mucosal distortion as seen in the presence of a vascular malformation.

\section{Conclusion}

The pathological spectrum of small bowel disease is wide, and other than the pathognomonic features of Crohn's disease, all abnormalities have a differential diagnosis that should be interpreted in conjunction with the patient's clinical history and consultation with the referring doctor.

The report should be kept as simple as possible with positive and negative findings reflecting the radiologist's understanding of the clinical problem. 